Trauma Berufskrankh 2004 - 6 [Suppl 3] : S334-S338 DOI 10.1007/s10039-003-0746-8

Online publiziert: 14. Juni 2003

(c) Springer-Verlag 2003
K.-H. Thielke - F. Barth - V. Echtermeyer

Unfallchirurgische Klinik, Klinikum Minden

\title{
Akromioklavikulargelenk- verletzungen
}

\section{Operative Möglichkeiten und Resultate}

ie Problematik des AC-Gelenk-Schadens, ob frisch oder chronisch, liegt in

- der schmerzhaften Bewegungseinschränkung,

- der fehlenden Belastbarkeit der Schulter und

- bei schlanken Patienten in der Deformität des Schulterprofils.

\section{Ursachen}

Die häufigste Ursache der Schultereckgelenkverletzung ist der Sport- und Freizeitunfall mit einem direkten Anpralltrauma auf die Schulterspitze bei adduziertem Arm. Seltener sind ein direktes Trauma auf den ausgestreckten Arm oder Sturz auf den Ellenbogen. In der Häufigkeit folgen Verkehrsunfälle - darunter v. a. Zweiradunfälle - und Arbeitsunfälle.

\section{Diagnose}

Eine exakt erhobene Anamnese ist richtungsweisend, die vermutete Diagnose lässt sich bereits durch eine klinische Untersuchung erhärten. Inspektorisch zeigt sich eine schmerzhafte Schonhaltung des Arms. Bei der frischen Verletzung wird der Arm meist am Körper gehalten und von der Hand der Gegenseite im Ellenbogenbereich unterstützt. So wird das schmerzhafte Eigengewicht des Arms neutralisiert. Vielfach zeigen sich Abschürfungen und Prellmarken im Bereich des Schultereckgelenks.
Das so genannte „Klaviertastenphänomen" lässt sich palpatorisch nach einem frischen Trauma schmerzbedingt häufig nicht auslösen. Schmerzärmer ist vielmehr die schon vom Patienten selbst durchgeführte Anhebung des SchulterArm-Komplexes zur Reposition. Das aktive Bewegungsausmaß nach der NeutralNull-Methode ist weitgehend eingeschränkt. Passiv toleriert der Patient nur geringe Bewegungsausschläge.

Röntgenaufnahmen (• Abb. 1) exakt in 2 Ebenen dienen dem Frakturausschluss. Eine streng axillo-axillare Aufnahme ist obligat, um auch in der Horizontalebene die physiologische Gelenkstellung beurteilen zu können. Durch
Panoramaaufnahmen beider Schultergelenke mit zurückgezogenen Schultern und bilateraler Belastung von je 10-15 kg lässt sich die Diagnose bestätigen.

Eine bilaterale Sonographie der Rotatorenmanschette zum Ausschluss von Begleitverletzungen sowie des AC-Gelenks schließt sich an.

\section{Klassifikationen}

Am Akromioklavikulargelenk unterscheiden wir eine horizontale Stabilität, v. a. gesichert durch das Lig. acromioclaviculare und die Deltotrapezoidfaszie, von einer vertikalen Stabilität, für die die korakoklavikularen Bänder verantwortlich
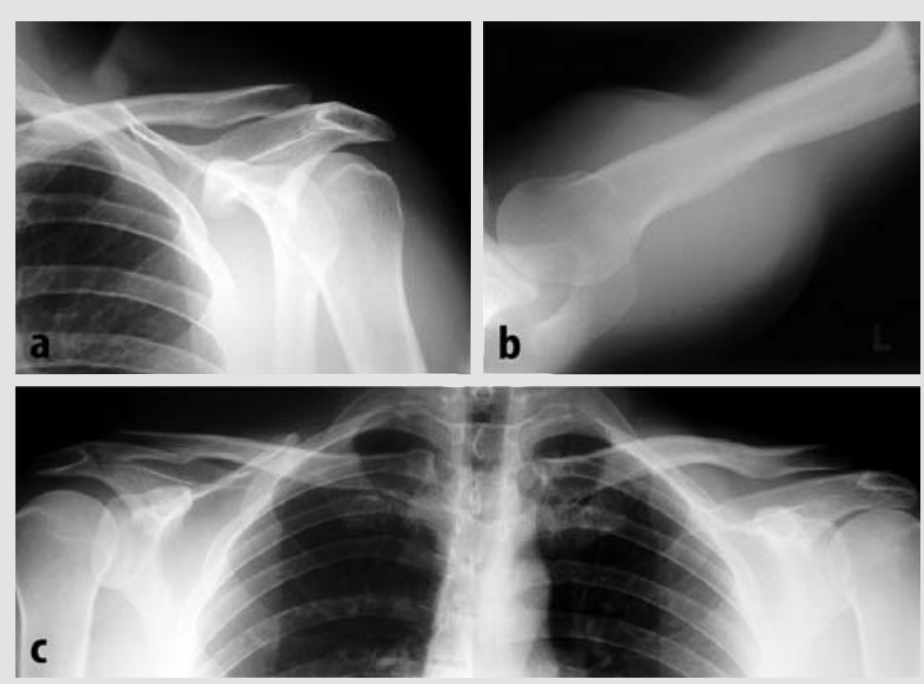

Abb. 1a-c $\Delta$ Standardröntgendiagnostik, Schulter exakt in 2 Ebenen, bei Verdacht auf AC-Gelenk-Sprengung: Panoramaaufnahmen beider Schultergelenke 

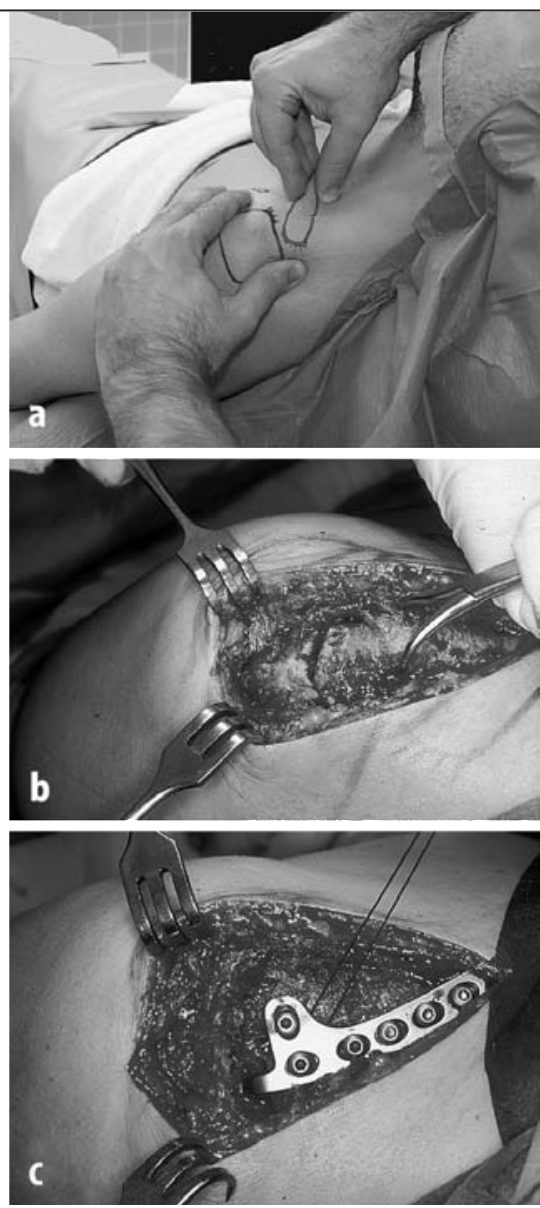

Abb. 2a-c $\Delta$ Lagerung zur Operation (Beachchair-Position) und Überprüfung der horizontalen Stabilität (a), Darstellung des AC-Gelenks, Fassen des lateralen Klavikulaendes mit einer Weller-Zange und Reposition (b), Anlagerung und Fixation der Hakenplatte sowie Naht der Gelenkkapsel bzw. des Lig. acromioclaviculare (c)

sind. Die Einteilungen der Verletzungen des AC-Gelenks bzw. deren Klassifikationen berücksichtigen das Ausmaß der Läsionen von Bändern, Faszien und Muskeln sowie die daraus resultierende horizontale und vertikale Instabilität. Tossy et al. [23] beschrieben 1963 eine Klassifikation der AC-Gelenk-Verletzungen vom Typ I-III, diese wurde 1967 im Wesentlichen von Allman [1] bestätigt. Rockwood et al. [18] erweiterten diese Klassifikation 1975 um 3 weitere Typen, wobei die Typen I-III identisch sind:

- Typ I nach Rockwood

Zerrung des AC-Gelenks bei intakten Bandverhältnissen

- Typ II nach Rockwood

Subluxation mit Zerreißung des AC-

Gelenks, aber intakten korakoklavi-

kularen Bändern

Trauma Berufskrankh 2004 - 6 [Suppl 3] : S334-S338

DOI 10.1007/s10039-003-0746-8

C Springer-Verlag 2003

\section{K.-H. Thielke $\cdot$ F. Barth $\cdot$ V. Echtermeyer}

\section{Akromioklavikulargelenkverletzungen. Operative Möglichkeiten und Resultate}

Zusammenfassung

Die Therapie der Akromioklavikulargelenksprengung, ob konservativ oder operativ, wird unterschiedlich definiert. Als Operationsindikationen werden neben den höhergradigen Verletzungstypen Rockwood IV, V und VI zumeist Sportler und körperlich schwer arbeitende Patienten mit einer Rockwood-II- oder -III-Verletzung genannt. Zum einen soll dadurch möglichen Spätschäden vorgebeugt, zum anderen aber die kosmetisch störende Fehlstellung beseitigt werden. Eine weitere Unsicherheit - hinsichtlich der Operationsmethode - spiegelt sich in der enormen Vielzahl von operativen Behandlungsvorschlägen zur Versorgung der Akromioklavikulargelenksprengung wider. In dem vorliegenden Beitrag sollen zunächst die Diagnostik und die Einteilung der Schultereckgelenksprengung sowie die geschichtliche Entwicklung der Versorgungsmöglichkeiten besprochen und anschließend ein differenziertes Behandlungskonzept zur Diskussion gestellt werden.

Schlüsselwörter

AC-Gelenk · Klassifikation · Operation · Outcome

\section{Acromioclavicular joint injuries. Surgical treatment and results}

\section{Abstract}

There is some controversy over the treatment of acromioclavicular injuries. The use of the Rockwood classification as the basis for decisions on whether operative or nonoperative treatment is indicated is discussed critically, and the authors' preferred operative technique is described and illustrated by examples. We treat injuries classified according to the Rockwood classification as types I and II with conservative methods. In type III injuries the patient's age, job and free time activities determine whether or not surgery is indicated. In the case of type IV or type VI injuries we always perform temporary internal fixation of the acromioclavicular joint, using transarticular K-wire fixation or hookplate osteosynthesis. Satisfactory results of both operative and nonoperative treatment are reported in the literature. The authors' own results are presented.

Keywords

Acromioclavicular joint · Classification · Surgical treatment $\cdot$ Outcome 
- Typ III nach Rockwood Lateraler Klavikulahochstand aufgrund einer zusätzlichen Zerreißung des Lig. coracoclaviculare; unter Belastung resultiert ein Hochstand der Klavikula um Schaftbreite

- Typ IV nach Rockwood Horizontale Instabilität, wobei die laterale Klavikula nach dorsal disloziert ist

- Typ V nach Rockwood Klavikulahochstand von bis zu 3 facher Schaftbreite aufgrund einer Verletzung des M. trapezius dorsal und des M. deltoideus ventral der Klavikula

- Typ VI nach Rockwood Seltene subakromiale oder subkorakoidale Verrenkung des lateralen Klavikulaendes

\section{Therapie}

\section{Systematik der Behandlungsverfahren}

Verletzungen Typ I und II

nach Rockwood

Verletzungen des Typs I und II nach Rockwood behandeln wir rein konservativ mit kurzfristiger Ruhigstellung und begleitender symptomatischer und antiphlogistischer Therapie. Eine frühfunktionelle Nachbehandlung ist indiziert. Der Unfallverletzte sollte stärkere Belastungen sowie Kontaktsportarten für mindestens 6 Wochen meiden.

\section{Verletzungen Typ III nach Rockwood}

Bei Typ-III-Verletzungen sollte die Indikation zum Therapieverfahren sorgfältig geprüft werden [2]. Sie wird u. a. vom Alter, Berufsbild und der sportlichen Aktivität des Patienten abhängig gemacht.

Bei konservativer Behandlung von Rockwood-III-Verletzungen kommt zunächst eine Immobilisierung im Gilchrist- oder Tape-Verband in Betracht. Nachfolgende Krankengymnastik bzw. Anleitungen zu Eigenübungen dienen dem Erwerb der freien Beweglichkeit.

Unbefriedigende Resultate werden üblicherweise nach 6 Monaten manifest und geben Anlass zur operativen Korrektur [21].
Verletzungen Typ IV-VI nach Rockwood

Die seltener Typen IV-VI erfordern in der Regel ein operatives Vorgehen.

\section{Operationsmethoden}

Bereits 1861 hat Cooper den ersten Versuch einer operativen Versorgung des ACGelenks mittels Silberdraht beschrieben [24]. In den folgenden 150 Jahren sind über 200 Publikationen erschienen, in denen sich etwa 100 Autoren mit einer „neuen“ Technik bzw. „Modifikation“ beschäftigten. Im Wesentlichen lassen sich die Operationsverfahren in 6 Gruppen einteilen:

- Bei den intra-/transartikulären Techniken verwendet man KirschnerDrähte, welche das AC-Gelenk kreuzen [4].

- Die extraartikulären Techniken bedienen sich einer Schraubenosteosynthese [5, 9] oder einer Hakenplatte [19], welche korrekt angelegt mit einem extraartikulär liegenden Haken dorsal unter das Akromion greift und über die Platte auf der Klavikula diese in ihre korrekte Lage zurückführt. Ebenfalls zu den extraartikulären Techniken zählen Zuggurtungsosteosynthesen [13] sowie PDS-Kordel-Augmentationen [11, 16].

- Bei der Resektionsarthroplastik wird das laterale Klavikulaende reseziert, mit und ohne Fixierung der Klavikula an das Korakoid.

- Beim dynamischen Muskeltransfer werden der Ursprung des M. coracobrachialis und der kurze Kopf des M. bizeps mit einer Knochenschuppe am Korakoid abgemeißelt und auf der Klavikula fixiert.

- Weiterhin gibt es Kombinationsverfahren der genannten Techniken [7, $12,17]$. Hierbei werden die akromioklavikulare und korakoklavikulare Stabilisierung mit metallischen Implantaten oder Kunstbändern durchgeführt.

- Bei der Arthrodese zwischen Akromion und Klavikula wird ein Knochenblock interponiert.

\section{Literaturüberblick}

Die Literaturdurchsicht von Vergleichsstudien $[2,3,14,21]$ zeigt, dass meistens über kleine Fallzahlen konservativ und operativ behandelter Patienten mit überwiegend guten bis ausgezeichneten Resultaten berichtet wird.

Die Vorteile des operativ versorgten Kollektivs bestehen in einer korrekt wiederhergestellten Anatomie und guter Funktion. Nachteilig sind die hohe Komplikationsrate, die Notwendigkeit einer zweiten Operation zur Implantatentfernung und eine längere Rehabilitation. Nicht in allen Fällen bestand Schmerzfreiheit.

Die Vorteile der konservativen Behandlungen liegen im nicht notwendigen Krankenhausaufenthalt, der kurzen Rehabilitation und der guten Funktion [2]. Als Nachteil werden auch hier eine fragliche Schmerzfreiheit sowie ein kosmetisch störendes Schulterprofil genannt.

Die Domäne der primär konservativen Behandlungen sind die Typen I und II nach Rockwood. Ebenso wird in der überwiegenden Mehrzahl der Patienten mit einer Typ-III-Verletzung die Indikation zur konservativen Behandlung gestellt. Andererseits ist die operative Therapie von Typ-IV-, -V- und -VI-Läsionen kaum Gegenstand von Diskussionen [2].

\section{Eigenes Therapieregime}

Zur Versorgung der AC-Gelenk-Sprengung wurden verschiedene operative Möglichkeiten beschrieben. Seit Mitte 2002 verwenden wir die Klavikulahakenplatte zur Versorgung der lateralen Klavikulafrakturen sowie auch der ACGelenk-Verletzungen. Das primär bei uns durchgeführte Therapieregime mit einer Zuggurtungsosteosynthese haben wir aufgrund nicht ausreichend guter Behandlungserfolge verlassen.

Nach Indikationsstellung zur operativen Versorgung mittels Hakenplatte wird der Patient in Beach-chair-Position gelagert, und die knöchernen Bezugspunkte werden angezeichnet ( $\bullet$ Abb. 2a). Über dem lateralen Ende der Klavikula wird bis etwa zur Akromionspitze inzidiert. Die Plattenlage wird präpariert (• Abb. 2b) und der Haken dorsal des AC-Gelenks 


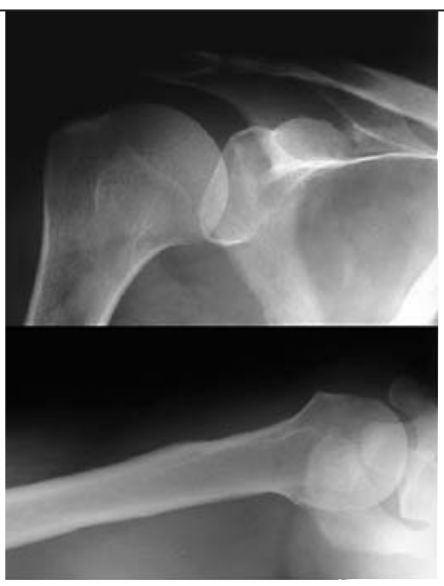

a April 2002

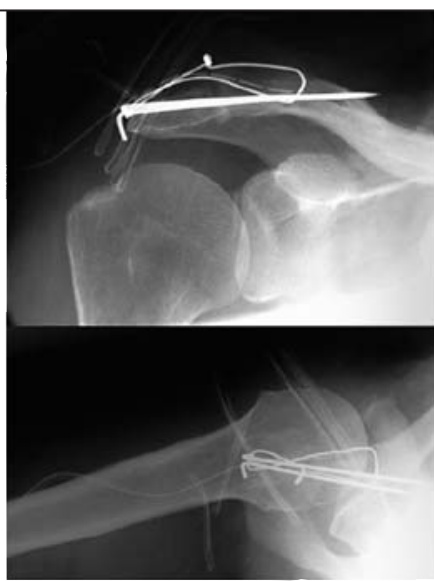

b Juni 2002

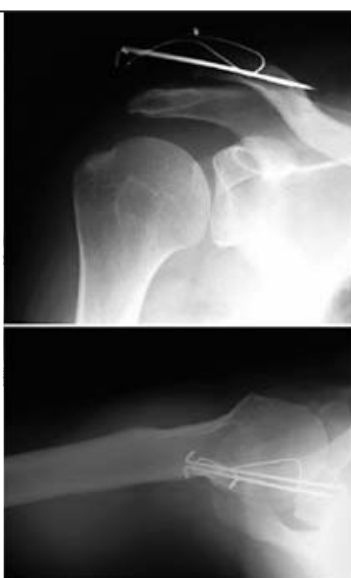

Juni 2002

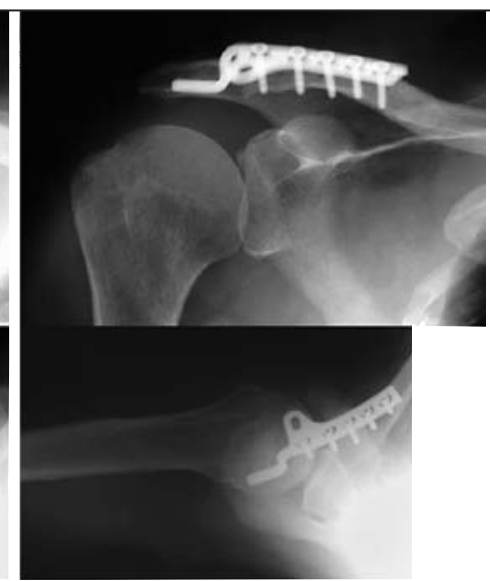

d Juli 2002

Abb. 3a-d $\Delta$ Rockwood-III-Verletzung der rechten Schulter bei 68-jährigem Patienten, a April 2002, b Juni 2002, c Juni 2002, d Juli 2002, Erläuterung s. Text

unter das Akromion eingeführt. Nach provisorischer Fixation der Platte am lateralen Klavikulaende und Überprüfung der Stabilität durch Zug am Arm werden die Plattenlöcher mit Kortikalisschraubenbesetzt (•Abb. 2c). Anschließend wird die Wunde schichtweise verschlossen. Eine Ruhigstellung wird nur für 2 Tage durchgeführt. Es schließt sich eine frühfunktionelle Nachbehandlung mit einer Limitierung der Abduktion bis $90^{\circ}$ an. Nach Ablauf von 3 Monaten sollte eine Metallentfernung durchgeführt werden.

\section{Komplikationsmöglichkeiten}

Anhand eines Fallbeispiels (• Abb. 3) können die bestehenden Risiken und Komplikationsmöglichkeiten der operativen Therapie demonstriert werden:

Ein 68-jähriger Patient zog sich im Rahmen eines Sturzereignisses neben einer Rippenserienfraktur und einer Skapulahalsfraktur eine Rockwood-III-Verletzung der rechten Schulter zu. Zunächst war eine konservative Behandlungsoption gegeben. Wegen des deutlichen Klavikulahochstands, fortbestehender Instabilität und persistierenden Beschwerden entschieden wir uns 2 Monate später zu einer operativen Therapie der AC-GelenkLuxation. Nach Zuggurtungsosteosynthese zeigte sich im frühen stationären Verlauf ein Implantatversagen mit Indikationsstellung zur Revision. Die Hakenplattenosteosynthese wurde komplikationslos mit nachfolgend unauffälligem
Verlauf durchgeführt. Nach initialer 2-tägiger Ruhigstellung erfolgten eine intensive krankengymnastische Übungsbehandlung und ein frühfunktionelles Nachbehandlungsregime. Eine Abduktion über $90^{\circ}$ wurde gemieden. Erst nach 6 Monaten wurde eine Metallentfernung durchgeführt. Dabei zeigte sich nativradiologisch eine durch den Haken bedingte sekundäre Arrosion unter dem Schulterdach. Diese war jedoch ohne Krankheitswert. Der Patient war subjektiv beschwerdefrei.

\section{Ergebnisse}

Bei einem jährlichen Aufkommen von etwa 10 operativen Stabilisierungen des AC-Gelenks in unserer Klinik sind Langzeit- und Kontrollergebisse schwer zu erheben und zu vergleichen. Mit einer Inzidenz von 3-4 Fällen pro 100.000 Einwohner und Jahr handelt es sich bei der Schultereckgelenksprengung um eine seltene Verletzung, die einer operativen Therapie zugeführt wird. Ähnlich verhält es sich mit den Veröffentlichungen im Literaturüberblick, wo verschiedene Verfahren und Methoden zur operativen Versorgung der AC-Gelenk-Sprengung beschrieben sind und anhand von nur kleinen Fallzahl (<50 Fälle) nachuntersucht wurden.

Bis 1997 behandelten wir die ACGelenk-Sprengung vorwiegend operativ [20]. Im Rahmen einer retrospektiven Nachuntersuchungsstudie (1988-1995) an 94 Patienten mit vorwiegend Rockwood-
II- und -III-Verletzungen (96,8\%) zeigte sich eine hohe Komplikationsrate von $32,3 \%$. Der Constant-Score [6] erbrachte 91,9 Punkte im Durchschnitt aller 62 nachuntersuchten Patienten bei einem mittleren Nachuntersuchungszeitraum von 62 Monaten. Seither werden AC-GelenkSprengungen Typ Rockwood II-III zur Vermeidung der operativen Komplikationen in unserer Klinik in ersten Linie konservativ behandelt.

Höhergradige Schultereckgelenkssprengungen sollten operativ behandelt werden. Die Zuggurtungsosteosynthese war bis Mai 2002 das Standardverfahren. Seither verwenden wir die Hakenplatte. Bisher liegen Frühergebnisse von 9 Patienten nach Hakenplattenosteosynthese vor. Dabei sind allesamt gute bis ausgezeichnete Ergebnisse zu verzeichnen gewesen. Einem größeren Patientenkollektiv bleibt es vorbehalten, ob sich die Hakenplatte etablieren und dem Literaturvergleich standhalten wird.

\section{Diskussion}

Schon früh wurde über eine chirurgische Behandlungsmöglichkeit der AC-GelenkSprengungen nachgedacht. Die erste operative Versorgung führte Cooper $1861 \mathrm{mit}$ einem Silberdraht durch [24]. Noch heute gebräuchliche Verfahren sind die Stabilisierung des Gelenks durch KirschnerDrähte mit und ohne Cerclage [20], mit unterschiedlichen Akromionplatten $[7,19]$ 
oder eine korakoklavikuläre Stellschraube [5].

Bei der aufwändigeren extraartikulären Plattenfixierung können Probleme im Bereich der deckenden Weichteile entstehen. Außerdem besteht die Gefahr eines subakromialen Impingementsyndroms. Die dynamische intraartikuläre Methode der Transfixation mit Spickdrähten und Cerclage führt neben unvermeidlichen Verletzungen der Gelenkflächen häufig zu Implantatlockerungen und Brüchen. Dies trifft auch für die korakoklavikulare Stellschraube zu. Bei allen zuletzt genannten Stabilisierungen muss der Patient einen Zweiteingriff zur Implantatentfernung in Kauf nehmen. In jüngster Vergangenheit kommt aufgrund dieser Nachteile der PDS-Kordel-Augmentation eine immer größere Bedeutung zu $[11,16]$. Die Vorteile der operativen Versorgung werden übereinstimmend in einem wiederhergestelltem Schulterprofil sowie einem guten funktionellen Ergebnis gesehen.

Jedoch zeigt auch die rein konservative Behandlung der Schultereckgelenksprengung im Literaturvergleich in etwa 90\% der Fälle ein gutes oder sehr gutes Ergebnis [8, 14, 15, 21]. Insbesondere bei Rockwood-III-Verletzungen ist eine Operationsindikation streng zu stellen.

Bei alltäglicher Beanspruchung des Schultergelenks scheint die konservative Behandlung nicht mit einer schlechteren Gebrauchsfähigkeit der betroffenen Extremität einherzugehen [10, 22]. Bedenkt man außerdem, dass bei etwa $30-40 \%$ der operierten Patienten unabhängig vom Operationsverfahren mit Repositionsverlusten bis hin zur vollständigen Reluxation im AC-Gelenk gerechnet werden muss, zwingt dies zu einer sehr sorgfältigen und engen Indikationsstellung sowie einer offenen Aufklärung des Patienten.

Die Indikation zur Operation wird in unserer Klinik - neben den Verletzungstypen IV-VI - bei Patienten mit einer Typ-III-Verletzung gesehen, die schwer körperlich arbeiten oder sportlich besonders ambitioniert sind.

\section{Hinweise für die Praxis}

\section{Die Therapie der AC-Gelenk-Verletzung ist patientenorientiert zu stellen. Konservativ sollten Rockwood-I-,-II- und -III-Verletzungen}

therapiert werden. Nur in Ausnahmefällen ist bei diesen eine operative Therapie angezeigt. Bei Verletzungen Typ Rockwood IV, V, und VI ist die operative Versorgung als Therapie der Wahl anzusehen. Es existieren verschiedene operative Möglichkeiten. Ein gutes bis sehr gutes Outcome ist in über $90 \%$ der Fälle zu verzeichnen.

\section{Korrespondierender Autor}

Dr. K.-H.Thielke

Unfallchirurgische Klinik, Klinikum Minden, Friedrichstraße 17,32425 Minden,

E-Mail:Thielke.KH@t-online.de

\section{Literatur}

1. Allman FL (1967) Fractures and ligamentous injuries of the clavicle and it's articulation.J Bone Joint Surg Am 49-A: 774-784

2. Baethis $H$, Tingart M, Bouillon B, Tiling $T$ (2000) Konservative oder operative Therapie der Schultereckgelenkverletzung - was ist gesichert? Eine systematische Literaturanalyse nach Kriterien der"evidence based medicine". Chirurg 71: 1082-1089

3. Bannister CC, Wallace WA, Stableforth PG, Hutson MA (1989) The management of acute acromioclavicular dislocation. A randomised prospective controlled trial. J Bone Joint Surg Br 71-B: 848-850

4. Blatter G, Meier G (1990) Augmentation der korakoklavikulären Bandnaht. Unfallchirurg 93: 578-583

5. Bosworth BM (1941) Acromioclavicular separation: new method of repair. Surg Gynecol Obstet 73: 866871

6. Constant CR, Murley AHG (1987) A clinical method of functional assessment of the shoulder. Clin Orthop 214: 160-164

7. Folwaczny EK,Stuermer KM (2001) Stabilisierung des luxierten Schultereckgelenks mit Bandnaht und BalserPlatte. Operat Orthop Traumatol 13: 211-220

8. Fremerey RW, Lobenhoffer P, Ramacker K, Gerich T, Skutek M, Bosch U (2001) Akute AC-Gelenksprengung operative oder konservative Therapie? Unfallchirurg 104: 294-299

9. Freudenschuß B, Boszotta H, Helperstorfer W (1991) Ergebnisse nach operativer Stabilisierung des zerrissenen Schultereckgelenkes. Unfallchirurg 94: 95-98

10. Glick JM, Millburn LJ, Haggerty JF (1977) Dislocated acromioclavicular joint: follow-up study of 35 unreduced acromioclavicular dislocation. Am J Sports Med 5: $264-270$

11. Hessmann M, Gotzen L, Gehling H, Ruschenpohler D (1997) Ergebnisse nach Rekonstruktion der Schultereckgelenksprengung unter Verwendung von PDSBändern. Unfallchirurg 100: 193-197

12. Krüger-Franke M, Maurer T, Rosemeyer B (1993) Ergebnisse eines kombinierten Operationsverfahrens bei der kompletten AC-Gelenkluxation Tossy III. Unfallchirurg 96: 1-5

13. Kuner EH, Kleiser E, Lindenmeier HL (1979) Die acromio-claviculäre Luxation. Aktuelle Traumatol 8: 205-209

14. Larsen E, Bjerg-Nielsen A, Christensen P (1986) Conservative or surgical treatment of acromioclavicular dislocation. J Bone Joint Surg Am 68-A: 552-555

15. Phillips AM, Smart C, Groom AFG (1998) Acromioclavicular dislocation. Conservative or surgical therapy. Clin Orthop 353: 10-17
16. Probst A, Hegelmaier C (1992) Die Stabilisierung des verletzten Schultereckgelenkes durch PDS-Kordel. Aktuelle Traumatol 22: 61-64

17. Rehn J, Pingel P, Hierholzer G (1970) Zur operativen Behandlung der Verrenkung im Schultereckgelenk. Acta Chir Austriaca 1: 30-37

18. Rockwood CA, Young DC (1990) Disorders of the acromioclavicular joint. In: Rookwood CA, Matsen FA (eds) The shoulder, vol 1. Saunders, Philadelphia, pp 413-476

19. Schindler A, Schmid JP, Heyse C (1985) Temporäre Fixation mit der Hakenplatte nach Balser bei der Behandlung der frischen vollständigen Schultereckgelenkssprengung. Unfallchirurg 88: 533-540

20. Schmidt A, Lill H, Lange K, Echtermeyer V (1997) Schultereckgelenkverletzungen: Effiziente Therapie unter ökonomischen Aspekten. Langenbecks Arch Chir 114: 1262-1264

21. Taft T, Wilson FC, Oglesby JW (1987) Dislocation of the acromioclavicular joint. J Bone Joint Surg Am 69-A: 1045-1051

22. Tibone J, Sellers R, Tonino P (1992) Strength testing after third-degree acromioclavicular dislocations. Am Sports Med 20: 328-331

23. Tossy JD, Mead NC, Sigmond HM (1963) Acromioclavicular separations: useful and practical classification for treatment. Clin Orthop 28: 111-119

24. Usadel G (1940) Die Behandlung der Schultereckverrenkung mit Kopfwärtslagerung des Schlüsselbeins. Ergebn Chir 33: 387 